University of Nebraska - Lincoln

DigitalCommons@University of Nebraska - Lincoln

\title{
Thermodynamically coupled heat and mass flows in a reaction- transport system with external resistances
}

Yasar Demirel

University of Nebraska Lincoln, ydemirel2@unl.edu

Follow this and additional works at: https://digitalcommons.unl.edu/cbmedemirel

Part of the Chemical Engineering Commons

Demirel, Yasar, "Thermodynamically coupled heat and mass flows in a reaction-transport system with external resistances" (2009). Yasar Demirel Publications. 2.

https://digitalcommons.unl.edu/cbmedemirel/2

This Article is brought to you for free and open access by the Chemical and Biomolecular Research Papers -- Faculty Authors Series at DigitalCommons@University of Nebraska - Lincoln. It has been accepted for inclusion in Yasar Demirel Publications by an authorized administrator of DigitalCommons@University of Nebraska - Lincoln. 


\title{
Thermodynamically coupled heat and mass flows in a reaction-transport system with external resistances
}

\author{
Yaşar Demirel \\ Department of Chemical and Biomolecular Engineering, University of Nebraska-Lincoln, \\ Lincoln, NE 68588, USA; tel 402 472-2745, fax 402 472-6989, email ydemirel2@unl.edu
}

\begin{abstract}
Considerable work has been published on mathematically coupled nonlinear differential equations by neglecting thermodynamic coupling between heat and mass flows in reaction-transport systems. The thermodynamic coupling refers that a flow occurs without or against its primary thermodynamic driving force, which may be a gradient of temperature, or chemical potential, or reaction affinity. This study presents the modeling of thermodynamically coupled heat and mass flows of two components in a reaction-transport system with external heat and mass transfer resistances. The modeling equations are based on the linear nonequilibrium thermodynamics approach by assuming that the system is in the vicinity of global equilibrium. The modeling equations lead to unique definitions of thermodynamic coupling (cross) coefficients between heat and mass flows in terms of kinetic parameters and transport coefficients. These newly defined parameters need to be determined to describe coupled reaction-transport systems. Some representative numerical solutions obtained by MATLAB illustrate the effect of thermodynamic coupling coefficients on the change of temperature and mass concentrations in time and space.
\end{abstract}

Keywords: balance equations, reaction-transport systems, thermodynamic coupling, nonequilibrium thermodynamics, heat of transport

\section{Introduction}

Considerable work has been published on mathematically coupled nonlinear differential equations for reaction-transport systems in porous catalyst by neglecting the thermodynamic coupling. Here the thermodynamic coupling refers that a flow (i.e. heat or mass flow or a reaction velocity) occurs without its primary thermodynamic driving force, or opposite to the direction imposed by its primary driving force. The principles of thermodynamics allow the progress of a process without or against its primary driving force only if this process is coupled with another spontaneous process. This is consistent with the statement of second law, which states that a finite amount of organization may be purchased at the expense of a greater amount of disorganization in a series of coupled processes.

Thermodynamically coupled chemical reaction-transport systems control the behavior of many transport and rate processes in physical, chemical and biological systems, and require a through analysis accounting the induced flows by cross effects [1-9]. Many published work, including some recent ones [10-12], on reaction-diffusion systems mainly consider mathematically coupled nonlinear differential relationships. More than 50 years ago, Turing [13] demonstrated that a reaction-diffusion system with appropriate nonlinear kinetics can cause instability in a homogeneous steady state and generate stable concentration patterns. Also the thermodynamic coupling in the membranes of living cells plays major role in the respiratory electron transport leading to synthesizing ad- enosine triphosphate $[6,14,15]$. Another important thermodynamic coupling takes place between the hydrolysis of adenosine triphosphate and the molecular transport of substrates in active transport. The coupling between a scalar process of the hydrolysis and a vectorial process of the mass flow creates the molecular pumps responsible for uphill transport [1, 14, 15]. Therefore, incorporation of thermodynamic coupling into the modeling of reaction-diffusion systems, such as active transport, may be a vital step in describing these complex biochemical cycles.

Two previous studies presented the modeling equations and approximate solutions for reaction-transport systems with thermodynamic coupling between heat and mass flows [4] and between transport processes and chemical reaction [5] without external resistances. This study presents the modeling equations for thermodynamically coupled heat and mass flows in a three-component system with an elementary chemical reaction and with external transport resistances. Therefore, it is a through analysis accounting the cross effects as well as external effects. The modeling is based on the linear nonequilibrium thermodynamics (LNET) formulations by assuming that the system is in the vicinity of global equilibrium (GE). The LNET formulation does not require the detailed mechanism of the thermodynamic coupling $[6,15]$. The modeling equations have produced some unique parameters related to thermodynamic couplings between heat and mass flows. These parameters combine the kinetic parameters and transport coefficients and control the cross effects. Some representative solutions of 


\begin{tabular}{|c|c|c|c|}
\hline \multicolumn{4}{|c|}{ Nomenclature } \\
\hline$a_{\mathrm{i}}$ & parameters in Equation (37) & $t$ & time, $\mathrm{s}$ \\
\hline$A$ & chemical affinity, $\mathrm{J} / \mathrm{mol}$ & $T$ & temperature, $\mathrm{K}$ \\
\hline$c_{p}$ & specific heat capacity, J/(kg K) & $w_{i}$ & mass fraction of component $i$ \\
\hline $\mathrm{Da}$ & Damköhler number & $X^{l}$ & thermodynamic force \\
\hline$D_{S}$ & effective diffusion coefficient for the substrate $\mathrm{S}, \mathrm{m}^{2} / \mathrm{s}$ & $z$ & dimensionless distance \\
\hline$D_{D}$ & $\begin{array}{l}\text { coupling coefficient related to the Dufour effect, } \\
\mathrm{J} \mathrm{m}^{2} /(\mathrm{mol} \mathrm{s})\end{array}$ & \multicolumn{2}{|c|}{ Greek letters } \\
\hline \multirow{2}{*}{$D_{T}$} & coupling coefficient related to the thermal diffusion & \multirow{3}{*}{$\begin{array}{l}\beta \\
\beta_{S}^{\prime}\end{array}$} & thermicity group, dimensionless \\
\hline & (Soret) effect, $\mathrm{mol} /(\mathrm{m} \mathrm{s} \mathrm{K})$ & & thermicity group for thermodynamically coupled \\
\hline$E$ & activation energy of the chemical reaction, $\mathrm{J} / \mathrm{mol}$ & & processes, dimensionless \\
\hline$h$ & heat transfer coefficient, $\mathrm{J} /\left(\mathrm{m}^{2} \mathrm{~K}\right)$ & \multirow[t]{2}{*}{$\varepsilon$} & \multirow{2}{*}{$\begin{array}{l}\text { dimensionless parameter related to Soret effect in Equa- } \\
\text { tion (42) }\end{array}$} \\
\hline$h_{i}$ & partial enthalpy, $\mathrm{J} / \mathrm{kg}$ & & \\
\hline$\Delta H_{r}$ & reaction enthalpy, $\mathrm{J} / \mathrm{kg}$ & $\Phi$ & volumetric entropy generation rate, $\mathrm{W} /\left(\mathrm{m}^{3} \mathrm{~K}\right)$ \\
\hline$H^{E^{\prime}}$ & excess specific enthalpy, $\mathrm{J} / \mathrm{kg}$ & $r$ & Arrhenius group, dimensionless \\
\hline j & diffusive mass flux, $\mathrm{mol} /\left(\mathrm{m}^{2} \mathrm{~s}\right)$ & $\phi$ & dimensionless temperature, Equation (40) \\
\hline$J_{q}$ & conduction heat flux, $\mathrm{W} / \mathrm{m}^{2}$ & $\varphi$ & diffusivity ratios, Equation (42) \\
\hline$J_{r}^{q}$ & volumetric reaction rate, $\mathrm{mol} /\left(\mathrm{m}^{3} \mathrm{~s}\right)$ & $\mu$ & chemical potential, $\mathrm{J} / \mathrm{mol}$ \\
\hline$k$ & effective thermal conductivity, W/(m K) & $\theta$ & dimensionless composition, Equation (40) \\
\hline$k_{\mathrm{g}}$ & external mass transfer coefficient, $\mathrm{m} / \mathrm{s}$ & $\lambda$ & relation in Equation (16) \\
\hline$k_{v}^{g}$ & first order reaction rate constant, $1 / \mathrm{s}$ & $v$ & stoichiometric coefficient \\
\hline$k_{0}^{0}$ & frequency in the Arrhenius equation, $1 / \mathrm{s}$ & $\rho$ & density, $\mathrm{kg} / \mathrm{m}^{3}$ \\
\hline$L$ & characteristic half thickness, $\mathrm{m}$ & $\tau$ & dimensionless time \\
\hline Le & Lewis number & $\omega$ & dimensionless parameter related to Dufour effect in \\
\hline$L_{i k}$ & phenomenological coefficients & & Equation (42) \\
\hline$L_{q r}$ & $\begin{array}{l}\text { element of coupling coefficient between chemical reaction } \\
\text { and heat flow, } \mathrm{mol} \mathrm{K} /\left(\mathrm{m}^{2} \mathrm{~s}\right)\end{array}$ & & \\
\hline$L_{i r}$ & element of coupling coefficient between chemical reaction & $\mathrm{D}$ & Dufour \\
\hline & and mass flow of component $i, \mathrm{~mol}^{2} \mathrm{~K} /\left(\mathrm{J} \mathrm{m}^{2} \mathrm{~s}\right)$ & eq & equilibrium \\
\hline$n$ & number of components & $\mathrm{P}$ & product \\
\hline$n r$ & number of chemical reactions & $\mathrm{q}$ & heat \\
\hline $\mathrm{Nu}$ & Nusselt number & $\mathrm{r}$ & reaction \\
\hline$Q_{i}^{*}$ & heats of transport for component $i, \mathrm{~kJ} / \mathrm{kg}$ & $\mathrm{s}$ & surface \\
\hline$R$ & gas constant, $\mathrm{J} /(\mathrm{mol} \mathrm{K})$ & $\mathrm{T}$ & thermal diffusion \\
\hline Sh & Sherwood number & 1 & \\
\hline
\end{tabular}

thermodynamically and mathematically coupled partial differential equations are presented to illustrate the effects of coupling on the behavior of temperature and mass concentrations in time and space.

\section{Modeling equations}

We consider a single porous catalyst pellet that catalyzes the elementary reaction $v_{S} S+v_{B} \mathrm{~B} \rightarrow v_{P} \mathrm{P}$ with a first order kinetics based on the $S$. The well known balance equations are

$$
\begin{aligned}
& \rho \frac{\partial w_{S}}{\partial t}=-\nabla \cdot \mathbf{j}_{S}+v_{S} J_{r} \\
& \rho \frac{\partial w_{B}}{\partial t}=-\nabla \cdot \mathbf{j}_{B}+v_{B} J_{r} \\
& \rho c_{p} \frac{\partial T}{\partial t}=-\nabla \cdot \mathbf{J}_{q}+\left(-\Delta H_{r}\right) J_{r}+\rho \sum_{i=1}^{2} \sum_{j=1}^{2} H_{j j}^{E} D_{j i} \nabla w_{i} \nabla w_{j}
\end{aligned}
$$

where

$$
\rho D_{i l}=\sum_{j=1}^{2} L_{i j} f_{j k} \mu_{k l}, \quad f_{j k}=\delta_{j k}+\frac{w_{k}}{w_{n}}, \quad \mu_{k l}=\left(\frac{\partial \mu_{k}}{\partial w_{l}}\right)_{T, P} \quad(i, k, l=1,2)
$$

and $\delta_{j k}$ is the Kronecker delta, $w_{i}$ is the mass fraction of component $i, \mathbf{j}_{i}$ the vector of mass flow of component $i, \mathbf{J}_{q}$ is the vector of reduced heat flow $\mathbf{J}_{q}=\mathbf{q}-\sum_{\mathbf{i}=1}^{\mathrm{n}} \mathbf{j}_{i} h_{i}, \mathbf{q}$ is the total heat flow, $h_{i}$ is the partial molar enthalpy of species $i$, and $\Delta H_{r}$ is the heat of reaction, $v_{i}$ is the stoichiometric coefficient, which is negative for reactants, $H_{i i}^{E}=\left(\partial^{2} H^{E} / \partial w_{i}^{2}\right)_{T, P},(i=1,2)$, and $H^{E}$ is the excess specific enthalpy or heat of mixing, and the parameters $D_{i j}$ are the diffusion coefficients. The reaction velocity $J_{r}$ in terms of frequency $k_{0}$ and activation energy $E$ for a first order elementary reaction is

$$
J_{r}=k_{0} \exp \left(-\frac{E}{R T}\right) \rho_{S}
$$

By using the Fick and Fourier laws in one-dimensional domain of $y$-direction and neglecting any thermodynamic couplings and excess enthalpy effects, Equations (1)-(3) become

$$
\begin{aligned}
& \rho \frac{\partial w_{S}}{\partial t}=\rho D_{S} \frac{\partial^{2} w_{S}}{\partial y^{2}}+v_{S} J_{r} \\
& \rho \frac{\partial w_{B}}{\partial t}=\rho D_{B} \frac{\partial^{2} w_{B}}{\partial y^{2}}+v_{B} J_{r} \\
& \rho c_{p} \frac{\partial T}{\partial t}=k \frac{\partial^{2} T}{\partial y^{2}}+\left(-\Delta H_{r}\right) J_{r}
\end{aligned}
$$

where $D_{i}$ is the effective diffusivity for component $i$, and $k$ the effective thermal conductivity. The initial and boundary conditions with external resistances are

$$
\begin{aligned}
& t=0, \quad w_{S}=w_{S 0^{\prime}} \quad w_{B}=w_{B 0^{\prime}} \quad T=T_{0} \\
& y= \pm L, \quad \rho \frac{\partial w_{S}}{\partial y}=\frac{k_{g S}}{D_{S}}\left(w_{S b}-w_{S S}\right) \\
& \rho \frac{\partial w_{B}}{\partial y}=\frac{k_{g B}}{D_{B}}\left(w_{B b}-w_{B S}\right), \quad \frac{\partial T}{\partial y}=\frac{h_{f}}{k}\left(T_{b}-T_{S}\right)
\end{aligned}
$$


$y=0, \partial w_{S} / \partial y=\partial w_{B} / \partial y=\partial T / \partial y=0$ (symmetry conditions) (10)

where $k_{g i}$ is the extra particle mass transfer coefficient for component $i$, and $h_{f}$ is the heat transfer coefficient, indices $b$ refers to bulk fluid conditions, and $L$ is the half thickness of the slab. Equation (9) represents the external mass and heat transfer resistances, respectively. Diffusion may reduce averaged rates relative to that obtained if the concentration was everywhere $w_{S s}$ and $w_{B s}$. This limitation is known as the effectiveness factor $[16,17]$.

\section{Phenomenological equations}

Reaction-transport systems represent open and nonequilibrium systems with thermodynamic forces of temperature gradient, concentration gradient, and affinity. For the chemical reaction-transport system considered, the local rate of energy dissipation $[3-6,12]$ due to local rate of entropy production $\Phi$ $\left(\Phi=\sum_{j} j_{i} X_{i}\right)$ is

$$
T \Phi=\mathbf{J}_{q} \cdot \nabla \ln T-\mathbf{j}_{S} \cdot\left(\nabla \mu_{S}\right)_{T, P}-\mathbf{j}_{B} \cdot\left(\nabla \mu_{B}\right)_{T, P}+J_{r} A \geq 0
$$

where $\left(\nabla \mu_{i}\right)_{T, P}=\sum_{\mathrm{i}=1}^{\mathrm{n}-1}\left(\partial \mu_{i} / \partial w_{i}\right) \nabla w_{i}, \mu_{i}$ is the chemical potential of species $i$, and $A$ is the affinity $\left(A=-\sum v_{i} \mu_{i}\right)$. Equation (11) consists of scalar processes of chemical reactions and vectorial processes of heat and mass flows, while it excludes pressure, viscous, electrical, and magnetic effects.

We have a linear relationship between the reaction velocity and the chemical affinity for an elementary reaction if $|A /(R T)| \ll 1[5,6,14,15]$

$$
J_{r}=L_{r r} A=\frac{k_{f} \rho w_{S, e q}}{R} A
$$

where $k_{f}$ is the forward reaction rate, and the coefficient $L_{r r}$ with the Arrhenius equation is defined by $L_{r r}=\left[\rho k_{0} \exp \left(-E_{f}\right]\right.$ $\left.R T) w_{S, e q}\right] / R$. Equation (12) indicates that the value of $L_{r r}$ is dependent on the rate constant and consequently on the equilibrium concentration $w_{S, e q}$ and the amount of chemical catalyst. Some selected biological pathways occur at near GE conditions [14], and for some chemical reactions the formalism of LNET can be used in wider ranges than usually expected [21].

Equation (11) identifies the independent conjugate flows $j_{i}$ and forces $X_{k}$ to be used in the linear phenomenological equations $j_{i}=\sum_{k} L_{i k} X_{k}$ when the system is in the vicinity of GE [5, 18-20]. For an $n$-component system and with $n r$-number of chemical reactions with mass flows relative to center of mass, the phenomenological equations for heat, mass, and reaction flows become

$$
\begin{aligned}
& -\mathbf{J}_{q}=L_{q q} \nabla \ln T+\sum_{j=1}^{n-1} \sum_{k=1}^{n-1} \sum_{l=1}^{n-1} L_{q j} f_{j k} \mu_{k l} \nabla w_{l}-\mathbf{L}_{q r} A \\
& -\mathbf{j}_{i}=L_{i q} \nabla \ln T+\sum_{j=1}^{n-1} \sum_{k=1}^{n-1} \sum_{l}^{n-1} L_{i j} f_{j k} \mu_{k l} \nabla w_{l}-\mathbf{L}_{i r} A \\
& -J_{r i}=\mathbf{L}_{r q} \cdot \nabla \ln T+\sum_{j=1}^{n-1} \sum_{k=1}^{n-1} \sum_{l=1}^{n-1} f_{j k} \mu_{k l} \mathbf{L}_{r j} \cdot \nabla w_{l}-\sum_{m}^{n r} L_{i m} A_{m}
\end{aligned}
$$

For the reaction-transport system with three components of S, B, and P, Equations (13)-(15) reduce to

$$
\begin{aligned}
-\mathbf{J}_{q}=L_{q q} \nabla \ln T & +\left(L_{q S} \lambda_{S S}+L_{q B} \lambda_{B S}\right) \nabla w_{S} \\
& +\left(L_{q S} \lambda_{S B}+L_{q B} \lambda_{B B}\right) \nabla w_{B}-\mathbf{L}_{q r} A \\
-\mathbf{j}_{S}=L_{S q} \nabla \ln T & +\left(L_{S S} \lambda_{S S}+L_{S B} \lambda_{B S}\right) \nabla w_{S} \\
& +\left(L_{S S} \lambda_{S B}+L_{S B} \lambda_{B B}\right) \nabla w_{B}-\mathbf{L}_{S r} A
\end{aligned}
$$

$$
\begin{aligned}
\mathbf{j}_{B}=L_{B q} \nabla \ln T & +\left(L_{B S} \lambda_{S S}+L_{B B} \lambda_{B S}\right) \nabla w_{S} \\
& +\left(L_{B S} \lambda_{S B}+L_{B B} \lambda_{B B}\right) \nabla w_{B}-\mathbf{L}_{B r} A \\
-J_{r}=\mathbf{L}_{r q} \cdot \nabla \ln T & +\left(\mathbf{L}_{r S} \lambda_{S S}+\mathbf{L}_{r B} \lambda_{B S}\right) \cdot \nabla w_{S} \\
& +\left(\mathbf{L}_{r S} \lambda_{S B}+\mathbf{L}_{r B} \lambda_{B B}\right) \cdot \nabla w_{B}-L_{r r} A
\end{aligned}
$$

where

$$
\lambda_{i j}=\sum_{k=1}^{2} f_{i k} \mu_{k j} .
$$

The coefficients $L_{i j}$ represent the phenomenological coefficients, which are related by various constraints, such as Onsager's reciprocity, Gibbs-Duhem equation at equilibrium, and the choice of reference frame for diffusivities. Some of the phenomenological coefficients $L_{i k}$ may be identified using Fick's, Fourier's, and the mass action laws [5]. The cross coefficients $\left(L_{S q}\right.$ or $\left.L_{q S}\right)$ may be represented by the Soret coefficient $\left(s_{T}\right)$, or the thermal diffusion coefficient $\left(D_{T}\right)$, which are related to each other by $s_{T} D_{S}=D_{T o}$. The Soret coefficient changes in the range $10^{-2}-10^{-3} 1 / \mathrm{K}$ for gases, nonelectrolytes, and electrolytes, however it might be larger for polymer solutions [6, 15]. We may define two new effective diffusion coefficients of $\left(D_{T}\right.$ and $\left.D_{D}\right)$ that are related to the thermal diffusion $D_{T}=L_{S q} T^{-2}$ and the Dufour effect $D_{D}=L_{q S} \lambda_{S} / T$. For $L_{q S}=L_{S q}$, we have $D_{D}=D_{T} T$ $\lambda_{S}$, which is proved experimentally [15]. For liquids, the diffusion coefficient $D$ is of the order of $10^{-5} \mathrm{~cm}^{2} / \mathrm{s}$, and the thermal diffusion coefficient $D_{T}$ is of the order of $10^{-8}-10^{-10} \mathrm{~cm}^{2} /(\mathrm{s} \mathrm{K})$. For gases, the order of magnitude for $D$ and $D_{T}$ is $10^{-1} \mathrm{~cm}^{2} / \mathrm{s}$, and $10^{-4}-10^{-6} \mathrm{~cm}^{2} /(\mathrm{s} \mathrm{K})$, respectively $[15,18-21]$.

Equations (16)-(19) can be modified with some transport coefficients $[4-6,18-20]$, and we have

$$
\begin{aligned}
& -\mathbf{J}_{q}=k \nabla T+\rho D_{D S} \nabla w_{S}+\rho D_{D B} \nabla w_{B}-\mathbf{L}_{q r} A \\
& -\mathbf{j}_{S}=D_{T S} \nabla T+\rho D_{S} \nabla w_{S}+\rho D_{S B} \nabla w_{B}-\mathbf{L}_{S r} A \\
& -\mathbf{j}_{B}=D_{T B} \nabla T+\rho D_{B S} \nabla w_{S}+\rho D_{B} \nabla w_{B}-\mathbf{L}_{B r} A \\
& -J r=\mathbf{L}_{r q}^{\prime} \cdot \nabla T+\mathbf{L}_{r S}^{\prime} \cdot \nabla w_{S}+\mathbf{L}_{r B}^{\prime} \cdot \nabla w_{B}-L_{r r} A
\end{aligned}
$$

where

$$
\begin{aligned}
& k=L_{q q} / T, \quad \rho D_{i l}=\sum_{j=1}^{2} L_{i j} \lambda_{j l}=\sum_{j=1}^{2} \sum_{k=1}^{2} L_{i j} f_{j k} \mu_{k l}, \\
& D_{D S}=Q_{S}^{*} D_{S S}+Q_{B}^{*} D_{B S}, \quad D_{D B}=Q_{S}^{*} D_{S B}+Q_{B}^{*} D_{B B} \quad D_{T S}=L_{S q} \frac{1}{T}, \\
& D_{T B}=L_{B q} \frac{1}{T}, \quad \mathbf{L}_{r S}^{\prime}=\mathbf{L}_{r S} \lambda_{S S}+\mathbf{L}_{r B} \lambda_{B S}, \quad \mathbf{L}_{r B}^{\prime}=\mathbf{L}_{r S} \lambda_{S B}+\mathbf{L}_{r B} \lambda_{B B}, \\
& \mathbf{L}_{r q}^{\prime}=\mathbf{L}_{r q} \frac{1}{T}
\end{aligned}
$$

Here we assumed that $D_{S}=D_{B}$ and $D_{S B}=D_{B S}$. These equations are based on the chain rule and the Gibbs-Duhem equation at constant temperature and pressure $\nabla_{T} \mu_{n}=-\sum_{j}^{n-1}\left(w_{i} / w_{n}\right) \nabla_{T}$ $\mu_{i} \cdot Q_{i}^{*}$ is the heat of transport defined by $Q_{i}^{*}=\left(J_{q} J_{i}\right) \nabla_{T=0, j_{k \neq j}=0^{\prime}}$ and is a measure of local heat exchange necessary to maintain isothermal conditions during diffusion of component $i$. The two independent heats of transport in terms of phenomenological coefficients are

$$
\begin{aligned}
& Q_{S}^{*}=\left(L_{q S} L_{B B}-L_{q B} L_{B S}\right) / U \\
& Q_{B}^{*}=\left(L_{q B} L_{S S}-L_{q S} L_{S B}\right) / U
\end{aligned}
$$

where

$$
U=L_{S S} L_{B B}-L_{S B} L_{B S}
$$

If we can control the temperature and concentration gradients, the coupling coefficients between the chemical reaction and the flows of mass and heat may be determined by the following relations

$$
\mathbf{L}_{r S}=\mathbf{L}_{S r}=\left(\frac{\mathbf{j}_{S}}{A}\right)_{\nabla w_{S}=0, \nabla w_{B}=0, \nabla T=0}=\left(\frac{\partial \mathbf{j}_{S}}{\partial A}\right)_{\nabla w_{S}, \nabla w_{B}, \nabla T} \cong\left(\frac{\Delta \mathbf{j}_{S}}{\Delta A}\right)_{\nabla w_{S}, \nabla w_{B}, \nabla T}
$$




$$
\begin{aligned}
& \mathbf{L}_{r B}=\mathbf{L}_{B r}=\left(\frac{\mathbf{j}_{B}}{A}\right)_{\nabla w_{S}=0, \nabla w_{B}=0, \nabla T=0}=\left(\frac{\partial \mathbf{j}_{B}}{\partial A}\right)_{\nabla w_{S}, \nabla w_{B}, \nabla T} \cong\left(\frac{\Delta \mathbf{j}_{B}}{\Delta A}\right)_{\nabla w_{S}, \nabla w_{B}, \nabla T} \\
& \mathbf{L}_{r q}=\mathbf{L}_{q r}=\left(\frac{\mathbf{J}_{q}}{A}\right)_{\nabla w_{S}=0, \nabla w_{B}=0, \nabla T=0}=\left(\frac{\partial \mathbf{J}_{q}}{\partial A}\right)_{\nabla w_{S}, \nabla w_{B}, \nabla T} \cong\left(\frac{\Delta \mathbf{J}_{q}}{\Delta A}\right)_{\nabla w_{S}, \nabla w_{B}, \nabla T}
\end{aligned}
$$

Equations (20)-(23) reduce to the following conductance matrix form of linear phenomenological equations

where

$$
-\mathbf{J}=\mathbf{L X}
$$

$$
\mathbf{J}=\left(\begin{array}{c}
J_{q} \\
j_{S} \\
j_{B} \\
J_{r}
\end{array}\right), \quad \mathbf{L}=\left[\begin{array}{llll}
L_{11}^{\prime} & L_{12}^{\prime} & L_{13}^{\prime} & L_{14}^{\prime} \\
L_{21}^{\prime} & L_{22}^{\prime} & L_{23}^{\prime} & L_{24}^{\prime} \\
L_{31}^{\prime} & L_{32}^{\prime} & L_{33}^{\prime} & L_{34}^{\prime} \\
L_{41}^{\prime} & L_{42}^{\prime} & L_{43}^{\prime} & L_{44}^{\prime}
\end{array}\right], \quad \mathbf{X}=\left(\begin{array}{c}
\nabla T \\
\nabla w_{S} \\
\nabla w_{B} \\
A
\end{array}\right)
$$

Onsager's reciprocal relations states that $L_{i j}^{\prime}=L_{j i}^{\prime}$ if $j_{i}$ and $j_{j}$ have the same parity under time reversal, and $L_{i j}^{\prime}=-L_{j i}^{\prime}$ if $j_{i}$ and $j_{j}$ have the opposite parity. In the absence of pertinent symmetries or invariances, all types of cross-couplings are possible and lead to nonvanishing cross coefficients $L_{i j}^{\prime} \neq 0(i \neq j)$. If the structure of the system is invariant with respect to some or all of the orthogonal transformations, then the invariance will eliminate certain cross-couplings and their cross-coefficients will vanish. If these symmetries are not exact then the corresponding cross-couplings would be weak and negligible.

As Equations (20)-(23) show, for the nonvanishing cross coefficients $L_{i j}^{\prime} \neq 0(i \neq j)$, all the forces contribute for each flow, and hence the thermodynamic couplings exist between vectorial processes of heat and mass flows, and between vectorial and scalar processes of reaction and transport. Coupling between vectorial and scalar processes is possible only in an anisotropic medium according to the Curie-Prigogine principle [15], which states that "a scalar thermodynamic force such as chemical affinity, which has the high symmetry of isotropy, cannot cause a diffusion flow, which has lower symmetry because of its directionality." Generally, irreversible processes of different tensorial character do not couple with each other in an isotropic medium. Therefore, the cross-coefficients between the chemical reaction and transport processes of heat and mass $\mathbf{L}_{S r^{\prime}}, \mathbf{L}_{r S^{\prime}}, \mathbf{L}_{q r^{\prime}}$ and $\mathbf{L}_{r q}$ would vanish in an isotropic medium, or would have vectorial character due to morphology of the interface, or due to compartmental structure causing an anisotropic character. For example, in active transport in biological cells, the hydrolysis of ATP is coupled with the flow of sodium ions outside of the cell. The flow direction is controlled by the structure of the membrane and thermodynamic coupling mechanisms in mitochondria. The medium may be locally isotropic, although it is not spatially homogenous. In this case, the coupling coefficients are associated with the whole system $[11,12]$.

\section{Thermodynamically coupled reaction-transport systems}

By substituting Equations (20)-(23) into Equations (1)-(3), we have $[5,6,20]$

$$
\begin{aligned}
\rho \frac{\partial w_{S}}{\partial t}= & \nabla \cdot\left(D_{T S} \nabla T+\rho D_{S} \nabla w_{S}+\rho D_{S B} \cdot \nabla w_{B}-\mathbf{L}_{S r} A\right) \\
& +\left(\mathbf{L}_{r q}^{\prime} \cdot \nabla T+\mathbf{L}_{r S}^{\prime} \cdot \nabla w_{S}+\mathbf{L}_{r B}^{\prime} \cdot \nabla w_{B}-L_{r r} A\right) \\
\rho \frac{\partial w_{B}}{\partial t}= & \nabla\left(D_{T B} \nabla T+\rho D_{B S} \nabla w_{S}+\rho D_{B} \nabla w_{B}-\mathbf{L}_{B r} A\right) \\
& +\left(\mathbf{L}_{r q}^{\prime} \cdot \nabla T+\mathbf{L}_{r S}^{\prime} \cdot \nabla w_{S}+\mathbf{L}_{r B}^{\prime} \cdot \nabla w_{B}-L_{r r} A\right)
\end{aligned}
$$

$$
\begin{aligned}
\rho c_{p} \frac{\partial T}{\partial t}= & \nabla \cdot\left(k \nabla T+\rho D_{D S} \nabla w_{S}+\rho D_{D B} \nabla w_{B}-\mathbf{L}_{q r} A\right) \\
& +\left(-\Delta H_{r}\right)\left(-\mathbf{L}_{r q}^{\prime} \cdot \nabla T-\mathbf{L}_{r S}^{\prime} \cdot \nabla w_{S}-\mathbf{L}_{r B}^{\prime} \cdot \nabla w_{B}+L_{r r} A\right) \\
& +\rho\left(H_{S S}^{E} D_{S S} \nabla^{2} w_{S}+\left(H_{B B}^{E} D_{B S}+H_{S S}^{E} D_{S B}\right) \nabla w_{S} \cdot \nabla w_{B}\right. \\
& \left.+H_{B B}^{E} D_{B B} \nabla^{2} w_{B}\right)
\end{aligned}
$$

Equations (27)-(29) are valid for systems containing no pressure gradients, no surface effects, and no gravitational or other external body forces. These relationships represent the mathematically and thermodynamically coupled chemical reaction-transport systems. The thermodynamic coupling consists the coupling between vectorial processes of transport (heat and mass flows) as well as between scalar (chemical reactions) and transport processes. Therefore, the effective transport coefficients become the elements of related effective transport coefficient tensors. When ideal mixing of components is considered, excess enthalpy will vanish in Equation (3) and Equation (29).

Equations (27)-(29) also represent the evolution equations in time and space for thermodynamically and mathematically coupled transport and chemical reaction systems. They allow the stability analysis to be performed to predict possible bifurcation in time and space depending upon the flows, forces, transport coefficients, and kinetic parameters beside the other controlling parameters, such as the distance from GE.

Equations (27)-(29) can be reduced to some specific coupled phenomena cases. If we neglect the thermodynamic coupling between chemical reaction and transport processes, all the cross-coefficients $\mathbf{L}_{S r^{\prime}} \mathbf{L}_{r S^{\prime}} \mathbf{L}_{B r^{\prime}} \mathbf{L}_{r B^{\prime}} \mathbf{L}_{q r^{\prime}}$ and $\mathbf{L}_{r q}$ vanish, and Equations (27)-(29) reduce to

$$
\begin{aligned}
& \rho \frac{\partial w_{S}}{\partial t}=\nabla \cdot\left(D_{T S} \nabla T+\rho D_{S} \nabla w_{S}+\rho D_{S B} \nabla w_{B}\right)-L_{r r} A \\
& \rho \frac{\partial w_{B}}{\partial t}=\nabla \cdot\left(D_{T B} \nabla T+\rho D_{B S} \nabla w_{S}+\rho D_{B} \nabla w_{B}\right)-L_{r r} A \\
& \rho c_{p} \frac{\partial T}{\partial t}=\nabla \cdot\left(k \nabla T+\rho D_{D S} \nabla w_{S}+\rho D_{D B} \nabla w_{B}\right)+\left(-\Delta H_{r}\right) L_{r r} A
\end{aligned}
$$

Here, Equations (30)-(32) neglect the excess enthalpy, $H^{E}$.

\subsection{Special case: one-dimensional reaction-transport in a simple slab}

For a one-dimensional transport with constant density $\rho$ in a simple slab geometry, Equations (30)-(32) become

$$
\begin{gathered}
\frac{\partial \rho_{S}}{\partial t}=D_{T S}\left(\frac{\partial^{2} T}{\partial y^{2}}\right)+D_{S}\left(\frac{\partial^{2} \rho_{S}}{\partial y^{2}}\right)+D_{S B}\left(\frac{\partial^{2} \rho_{B}}{\partial y^{2}}\right)-k_{0} \exp \left(-\frac{E}{R T}\right) \rho_{S} \\
\frac{\partial \rho_{B}}{\partial t}=D_{T B}\left(\frac{\partial^{2} T}{\partial y^{2}}\right)+D_{B S}\left(\frac{\partial^{2} \rho_{S}}{\partial y^{2}}\right)+D_{B}\left(\frac{\partial^{2} \rho_{B}}{\partial y^{2}}\right)-k_{0} \exp \left(-\frac{E}{R T}\right) \rho_{S} \\
\rho c_{p} \frac{\partial T}{\partial t}=k\left(\frac{\partial^{2} T}{\partial y^{2}}\right)+D_{D S}\left(\frac{\partial^{2} \rho_{S}}{\partial y^{2}}\right)+D_{D B}\left(\frac{\partial^{2} \rho_{B}}{\partial y^{2}}\right) \\
+\left(-\Delta H_{r}\right) k_{0} \exp \left(-\frac{E}{R T}\right) \rho_{S}
\end{gathered}
$$

The initial and boundary conditions are the same as in Equations (8)-(10).

\subsection{Maximum temperature difference}

By eliminating the reaction terms in Equations (33) and (35) at steady state, and integrating once from the pellet center $(L=0)$ to surface with the boundary conditions, we have 


$$
\begin{aligned}
& \left.\left(D_{S}+\frac{D_{D S}}{\left(-\Delta H_{r}\right)}\right) \frac{d \rho_{S}}{d y}\right|_{L}+\left.\left(D_{S B}+\frac{D_{D B}}{\left(-\Delta H_{r}\right)}\right) \frac{d \rho_{B}}{d y}\right|_{L} \\
& +\left.\left(D_{T S}+\frac{k}{\left(-\Delta H_{r}\right)}\right) \frac{d T}{d y}\right|_{L}=0=\frac{k_{g S}}{D_{S}}\left\{\left(D_{S}+\frac{D_{D S}}{\left(-\Delta H_{r}\right)}\right)\left(\rho_{S b}-\rho_{S S}\right)\right. \\
& \left.+\left(D_{S B}+\frac{D_{D B}}{\left(-\Delta H_{r}\right)}\right)\left(\rho_{B b}-\rho_{B S}\right)\right\}+\left(D_{T S}+\frac{k}{\left(-\Delta H_{r}\right)}\right) \frac{h_{f}}{k}\left(T_{b}-T_{S}\right)
\end{aligned}
$$

From the right-hand side, we have the temperature difference between surface and bulk fluid temperatures by assuming that $k_{g S}=k_{g B}=k_{g}$ and $k_{S}=k_{B}=k$

$$
T_{s}-T_{b}=\left(\frac{\mathrm{Sh}}{\mathrm{Nu}}\right)\left[a_{1}\left(\rho_{S b}-\rho_{S S}\right)+a_{2}\left(\rho_{B b}-\rho_{B S}\right)\right]
$$

where

$$
a_{1}=\frac{D_{S}\left(-\Delta H_{r}\right)+D_{D S}}{D_{T S}\left(-\Delta H_{r}\right)+k}, \quad a_{2}=\frac{D_{S B}\left(-\Delta H_{r}\right)+D_{D B}}{D_{T S}\left(-\Delta H_{r}\right)+k}
$$

and $\mathrm{Sh}$ and $\mathrm{Nu}$ are the Sherwood and Nusselt numbers, respectively

$$
\mathrm{Sh}=\frac{k_{\mathrm{g}} L}{D_{S}}, \quad \mathrm{Nu}=\frac{h_{f} L}{k}
$$

After the second integration of Equation (36) from the pellet center to surface, and some arrangements, the total temperature difference $\left(T-T_{b}\right)$ becomes

$$
T-T_{b}=a_{1}\left(\rho_{S s}-\rho_{S}\right)+a_{2}\left(\rho_{B s}-\rho_{B}\right)+\left(T_{s}-T_{b}\right)
$$

The first two terms of the right-hand side represent the internal temperature difference, while the third term is the external temperature difference. Substituting Equation (37) into Equation (38), we have

$$
\begin{aligned}
T-T_{b}= & a_{1}\left[\left(\rho_{S S}-\rho_{S}\right)+\frac{\mathrm{Sh}}{\mathrm{Nu}}\left(\rho_{S b}-\rho_{S S}\right)\right] \\
& +a_{2}\left[\left(\rho_{B S}-\rho_{B}\right)+\frac{\mathrm{Sh}}{\mathrm{Nu}}\left(\rho_{B b}-\rho_{B S}\right)\right]
\end{aligned}
$$

By multiplying the both side of Equation (39) by $\left(\rho_{S s} / T_{s}\right)$ and after arranging, we have

$$
\phi-\phi_{b}=\beta_{S}^{\prime}\left(1-\theta_{S}\right)+\beta_{B}^{\prime}\left(1-\theta_{B}\right)+\frac{\mathrm{Sh}}{\mathrm{Nu}}\left(\theta_{S b}-1\right)\left(\beta_{S}^{\prime}+\beta_{B}^{\prime}\right)
$$

where

$$
\begin{aligned}
& \phi=\frac{T}{T_{s}}, \quad \phi_{b}=\frac{T_{b}}{T_{s}}, \quad \theta_{S}=\frac{\rho_{S}}{\rho_{S S}}, \quad \theta_{B}=\frac{\rho_{B}}{\rho_{S S}}, \quad \theta_{S b}=\frac{\rho_{S b}}{\rho_{S S}}=\frac{\rho_{B b}}{\rho_{S S}}, \\
& \rho_{S S}=\rho_{B S}, \quad \beta_{S}^{\prime}=\left(\frac{D_{S}\left(-\Delta H_{r}\right)+D_{D S}}{D_{T S}\left(-\Delta H_{r}\right)+k}\right) \frac{\rho_{S S}}{T_{S}} \\
& \beta_{B}^{\prime}=\left(\frac{D_{S B}\left(-\Delta H_{r}\right)+D_{D B}}{D_{T S}\left(-\Delta H_{r}\right)+k}\right) \frac{\rho_{S S}}{T_{S}}
\end{aligned}
$$

Equation (40) is the relationships between the dimensionless temperature $\phi$ and compositions $\theta_{S}$ and $\theta_{B}$ at steady state and with thermodynamic couplings. The maximum temperature difference occurs when $\theta_{S}=0$ and $\theta_{B}=0$, and Equation (40) becomes

$$
\phi_{\max }-\phi_{b}=\left(1+\frac{\mathrm{Sh}}{\mathrm{Nu}}\left(\theta_{S b}-1\right)\right)\left(\beta_{S}^{\prime}+\beta_{B}^{\prime}\right)
$$

The value of $\beta_{i}^{\prime}$ is a measure of nonisothermal effects at surface conditions for component $i$ when heat and mass flow are thermodynamically coupled. Equation (41) contains the cross effects due to thermodynamic couplings as well as the external resistance effects on the maximum temperature difference in a catalyst pellet.

\section{Some representative solutions and discussions}

Equations (33)-(35) reduce to the following dimensionless forms

$$
\begin{aligned}
& \frac{\partial \theta_{S}}{\partial \tau}=\varepsilon_{S} \frac{\partial^{2} \phi}{\partial z^{2}}+\frac{\partial^{2} \theta_{S}}{\partial z^{2}}+\varphi_{S} \frac{\partial^{2} \theta_{B}}{\partial z^{2}}-\operatorname{Da}_{S} \theta_{S} \exp \left[\gamma\left(1-\frac{1}{\phi}\right)\right] \\
& \frac{\partial \theta_{B}}{\partial \tau}=\varepsilon_{B} \frac{\partial^{2} \phi}{\partial z^{2}}+\varphi_{B} \frac{\partial^{2} \theta_{S}}{\partial z^{2}}+\delta \frac{\partial^{2} \theta_{B}}{\partial z^{2}}-\operatorname{Da}_{S} \theta_{B} \exp \left[\gamma\left(1-\frac{1}{\phi}\right)\right] \\
& \frac{1}{\text { Le }} \frac{\partial \phi}{\partial \tau}=\frac{\partial^{2} \phi}{\partial z^{2}}+\omega_{S} \frac{\partial^{2} \theta_{S}}{\partial z^{2}}+\omega_{B} \frac{\partial^{2} \theta_{B}}{\partial z^{2}}+\mathrm{Da}_{S} \beta \theta_{S} \exp \left[\gamma\left(1-\frac{1}{\phi}\right)\right]
\end{aligned}
$$

\begin{tabular}{|c|c|c|c|c|}
\hline Parameters & $\begin{array}{l}\text { Parameters } \\
\text { used in } \\
\text { Figure } 1\end{array}$ & $\begin{array}{l}\text { Parameters } \\
\text { used in } \\
\text { Figure } 2\end{array}$ & $\begin{array}{l}\text { Lower } \\
\text { bound } \\
{[17]}\end{array}$ & $\begin{array}{r}\text { Upper } \\
\text { bound } \\
\text { [17] }\end{array}$ \\
\hline$\overline{\beta=\left(-\Delta H_{r}\right) D_{s} \rho_{S_{s}} /\left(k T_{s}\right)}$ & 0.1 & 0.1 & 0 (exothermic) & c) \\
\hline$r=E /\left(R T_{s}\right)$ & 10 & 10 & 0 & 60 \\
\hline $\mathrm{Le}=k_{e} /\left(\rho C_{p} D_{S}\right)$ & 0.01 & 0.01 & 0.001 & 100 \\
\hline $\mathrm{Sh}=k_{g} L / D_{S}$ & 5 & 5 & 0.1 & 5000 \\
\hline $\mathrm{Nu}=h_{f} L / k^{3}$ & 0.25 & 0.25 & 0.01 & 50 \\
\hline $\mathrm{Sh} / \mathrm{Nu}$ & 20 & 20 & 1 & 2000 \\
\hline $\mathrm{Da}_{S}=L^{2} k_{0} \exp \left[\left(E /\left(R T_{s}\right)\right] / D_{S}\right.$ & $D_{S} 0.01$ & 0.01 & 0.005 & 100 \\
\hline$\varepsilon_{S}=D_{T S} T_{S} / D_{S} \rho_{S S}$ & 0.0001 & 0.01 & & \\
\hline$\varepsilon_{B}=D_{T B} T_{S} / D_{S} \rho_{S S}$ & 0.0001 & 0.01 & & \\
\hline$\varphi_{S}=D_{S B} / D_{S}$ & 1 & 1 & & \\
\hline$\varphi_{B}=D_{B S} / D_{S}$ & 1 & 1 & & \\
\hline$\delta=D_{B} / D_{S}$ & 1 & 1 & & \\
\hline$\omega_{S}=D_{D S} \rho_{S S} / k T_{S}$ & 0.0001 & 0.01 & & \\
\hline$\omega_{B}=D_{D B} \rho_{S S} / k T_{s}$ & 0.0001 & 0.01 & & \\
\hline$\theta_{S b}=\rho_{S b} / \rho_{S S}$ & 1.1 & 1.1 & & \\
\hline$\theta_{B b}=\rho_{B b} / \rho_{S S}=\theta_{B b}$ & 1.1 & 1.1 & & \\
\hline$\phi_{b}=T_{b} / T_{s}$ & 0.98 & 0.98 & & \\
\hline
\end{tabular}

where

$$
\begin{aligned}
& z=\frac{y}{L}, \quad \tau=\frac{D_{S} t}{L^{2}}, \quad \gamma=\frac{E}{R T_{s}}, \quad \text { Le }=\frac{k_{e} / \rho C_{p}}{D_{S}}, \\
& \mathrm{Da}_{S}=\frac{L^{2} k_{0} \exp \left(E / R T_{S}\right)}{D_{S}}, \quad \beta=\frac{\left(-\Delta H_{r}\right) D_{S} \rho_{S S}}{k T_{S}}, \quad \varepsilon_{S}=\frac{D_{T S} T_{S}}{D_{S} \rho_{S s}}, \\
& \varepsilon_{B}=\frac{D_{T B} T_{S}}{D_{S} \rho_{S S}}, \quad \varphi_{S}=\frac{D_{S B}}{D_{S}}, \quad \varphi_{B}=\frac{D_{B S}}{D_{S}}, \quad \delta=\frac{D_{B}}{D_{S}}, \quad \omega_{S}=\frac{D_{D S} \rho_{S S}}{k T_{S}}, \\
& \omega_{B}=\frac{D_{D B} \rho_{S S}}{k T_{S}}
\end{aligned}
$$

The initial and boundary conditions become

$$
\begin{array}{lll}
\tau=0 & \theta_{S}=\theta_{S o} \quad \theta_{B}=\theta_{B o} & \phi=\phi_{0} \\
z= \pm 1, \tau>0 & \frac{\partial \theta_{S}}{\partial z}=\operatorname{Sh}\left(\theta_{S b}-1\right), & \frac{\partial \theta_{B}}{\partial z}=\operatorname{Sh}\left(\theta_{B b}-1\right) \\
& \frac{\partial \phi}{\partial z}=\operatorname{Nu}\left(\phi_{b}-1\right) & \\
z=0, \tau>0, & \frac{\partial \theta_{S}}{\partial z}=\frac{\partial \theta_{B}}{\partial z}=\frac{\partial \phi}{\partial z}=0
\end{array}
$$

Here the coefficients $\varepsilon_{S}, \varepsilon_{B}, \omega_{S^{\prime}} \omega_{B}$ are the cross effects representing the thermodynamic couplings between heat and mass flows of species $\mathrm{S}$ and $\mathrm{B}$, respectively. $\mathrm{Da}_{i}$ is the Damköhler number for component $i$, and measures the intrinsic rates of the reactions relative to that of the diffusions.

Equations (42)-(45) reduce to the following stationary equations with thermodynamically coupled heat and mass flows

$$
\varepsilon_{S} \frac{\partial^{2} \phi}{\partial z^{2}}+\frac{\partial^{2} \theta_{S}}{\partial z^{2}}+\varphi_{S} \frac{\partial^{2} \theta_{B}}{\partial z^{2}}-\operatorname{Da}_{S} \theta_{S} \exp \left[\gamma\left(1-\frac{1}{\phi}\right)\right]=0
$$

Table 1. Some transport, kinetic, surface, and external resistance parameters for the reaction-diffusion system with heat opeffects $[4,5,17]$. 
$\varepsilon_{B} \frac{\partial^{2} \phi}{\partial z^{2}}+\varphi_{B} \frac{\partial^{2} \theta_{S}}{\partial z^{2}}+\delta \frac{\partial^{2} \theta_{B}}{\partial z^{2}}-\operatorname{Da}_{S} \theta_{B} \exp \left[\gamma\left(1-\frac{1}{\phi}\right)\right]=0$

$\frac{\partial^{2} \phi}{\partial z^{2}}+\omega_{S} \frac{\partial^{2} \theta_{S}}{\partial z^{2}}+\omega_{B} \frac{\partial^{2} \theta_{B}}{\partial z^{2}}+\operatorname{Da}_{S} \beta \theta_{S} \exp \left[\gamma\left(1-\frac{1}{\phi}\right)\right]=0$

a

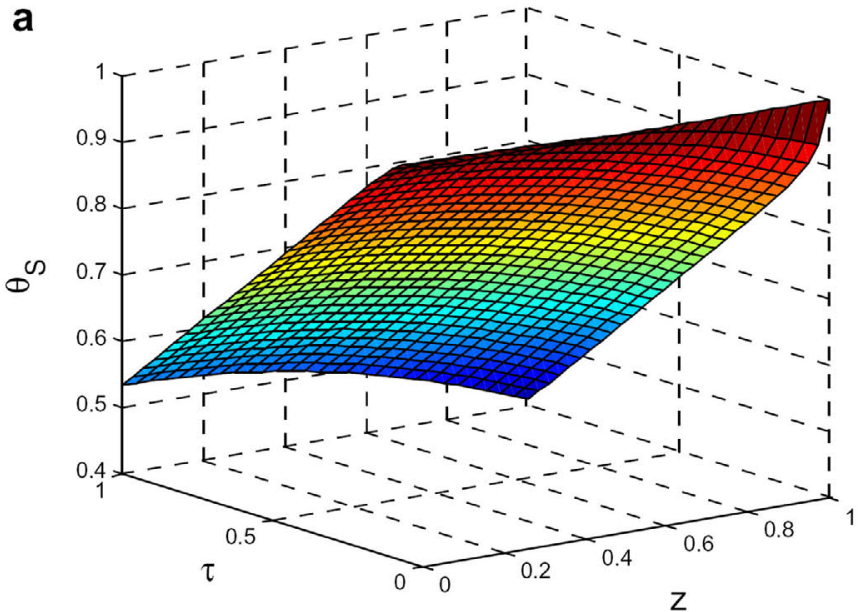

b
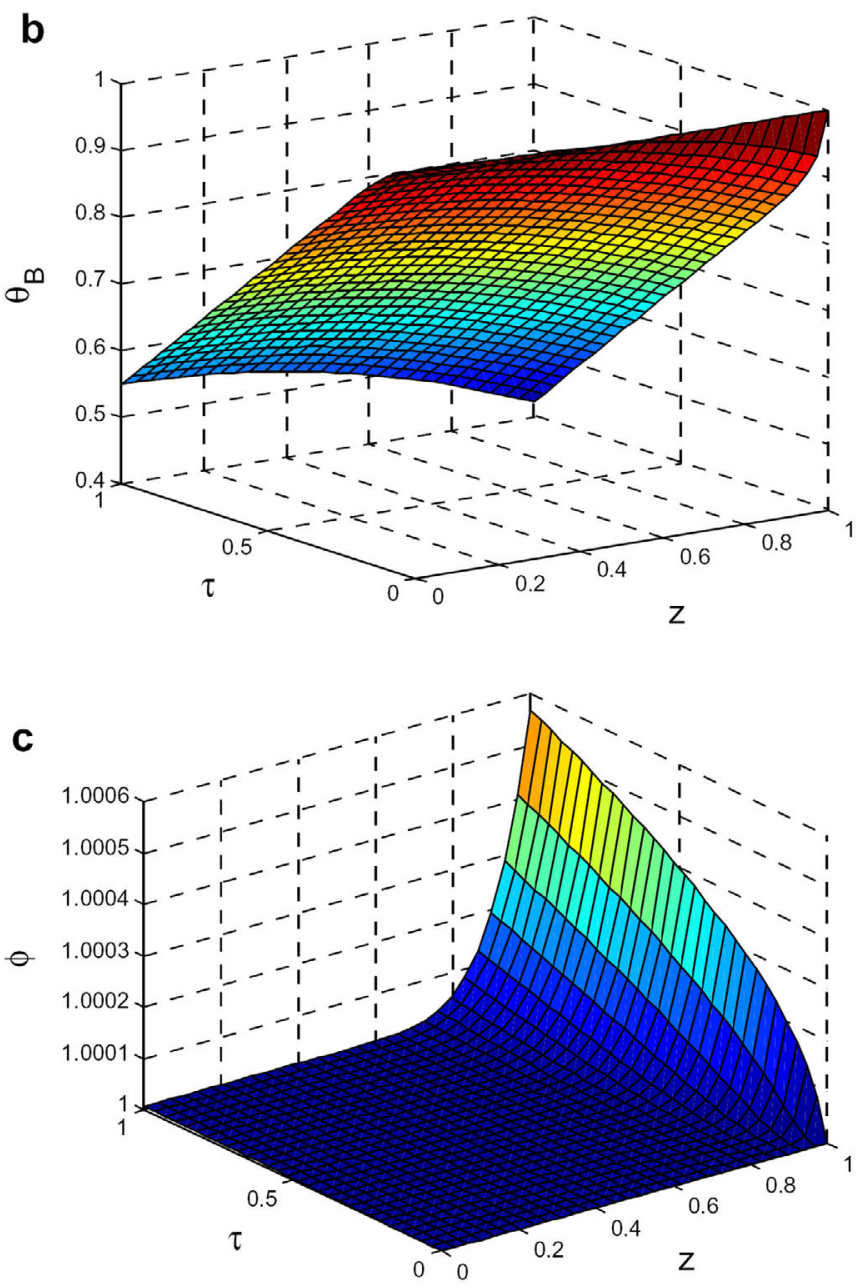

The MATLAB is used to solve the thermodynamically and mathematically coupled systems of Equations (42)-(45) by using the parameters listed in Table 1, which also lists lower and upper bounds for some of the parameters [17]. The chemical reaction is slow, as $\mathrm{Da}_{\mathrm{s}}=0.01$. Figure 1 and Figure 2 display the dynamic behavior of the mass concentration and temperature
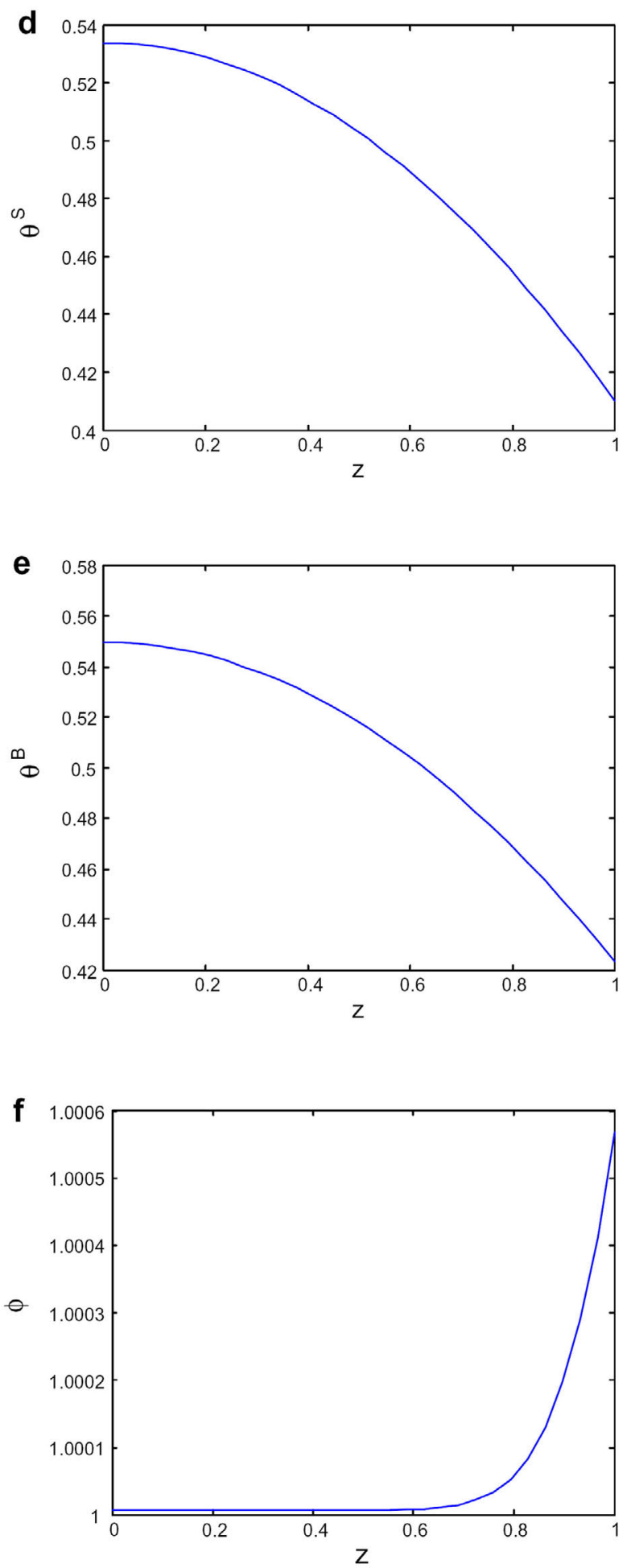

Figure 1. Dimensionless compositions and temperatures in time and space with $\varepsilon_{S}=\varepsilon_{B}=0.0001, \omega_{S}=\omega_{B}=0.0001$ and the parameters listed in Table 1: (a) behavior of component $\mathrm{S}$, (b) behavior of component $\mathrm{B}$, (c) behavior of temperature, (d) behavior of component $\mathrm{S}$ at $\tau=1$, (e) behavior of component $\mathrm{B}$ at $\tau=1$, (f) behavior of temperature at $\tau=1$. 
surfaces at two different set of cross coefficients $\varepsilon_{S}=\varepsilon_{B}=0.0001$ and $\varepsilon_{S}=\varepsilon_{B}=0.01$, and $\omega_{S}=\omega_{B}=0.0001$ and $\omega_{S}=\omega_{B}=0.01$ while keeping all the other parameters the same as listed in Table 1. Therefore, Figure 1 and Figure 2 display the effects of thermodynamic couplings between heat and mass flows, and compares such effects at two levels of cross coefficients of $\varepsilon$ and $\omega$. Here, for the purpose of comparison, it was assumed that $\varepsilon_{S}=\varepsilon_{B}=\omega_{S}=\omega_{B}$ at the upper and lower limits as well as
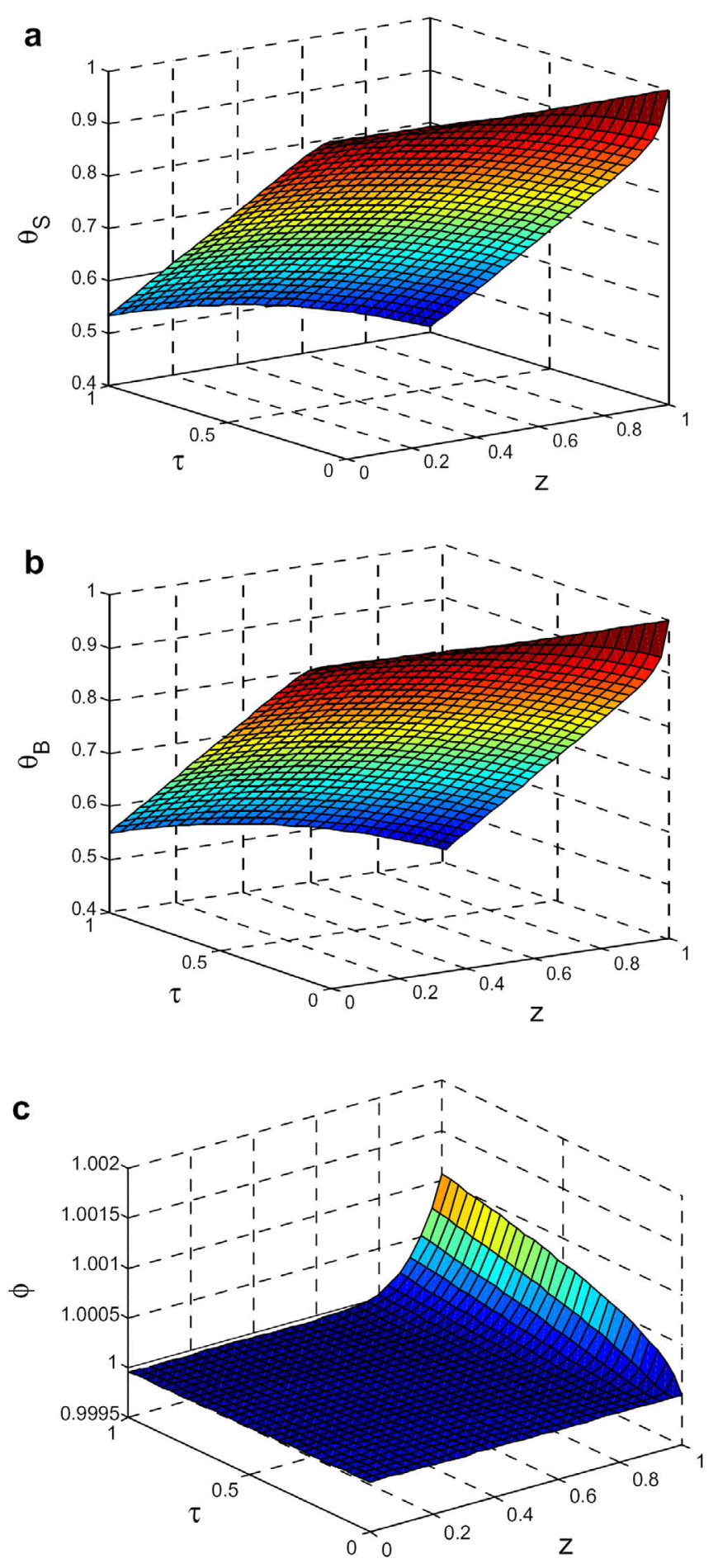

Figure 2. Dimensionless compositions and temperatures in time and space with $\varepsilon_{S}=\varepsilon_{B}=0.01, \omega_{S}=\omega_{B}=0.01$ and the parameters listed in Table 1 : (a) behavior of component $\mathrm{S}$, (b) behavior of component $\mathrm{B}$, (c) behavior of temperature, (d) behavior of component $\mathrm{S}$ at $\tau=1$, (e) behavior of component $\mathrm{B}$ at $\tau=1$, (f) behavior of temperature at $\tau=1$.

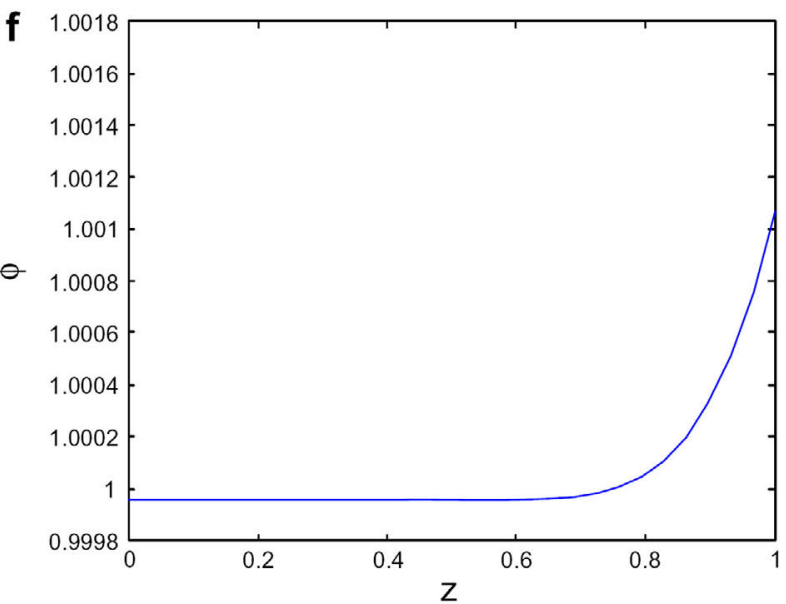

$D_{S}=D_{B}$; obviously the cross coefficients $\varepsilon$ and $\omega$ as well as the diffusion coefficients as $D_{S}$ and $D_{B}$ may be different. Figure 1 and Figure 2 display the mass concentrations and temperatures at the end point where the dimensionless time $\tau=1$.

The surfaces of temperatures and mass concentrations depend on the values of coefficients representing the thermodynamic couplings and the assigned values of other parameters. As Figure 1 and Figure 2c show, for the higher values of cross
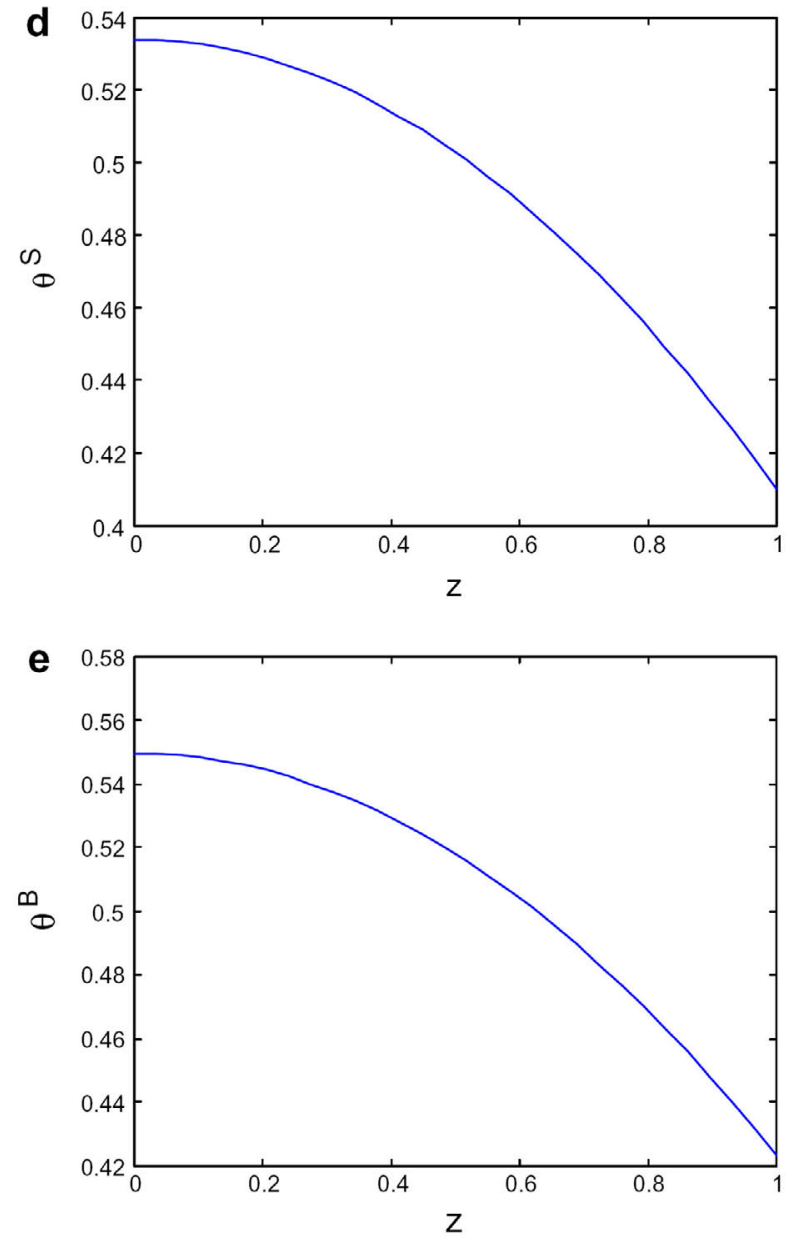
coefficients of $\varepsilon$, and $\omega$, the value of $\phi$ increases slightly (form 1.0006 approximately to 1.0011 ) and the nonequilibrium region (nonisothermal) shrinks slightly at $\tau=1$. The changes in the mass concentrations are marginal with the changes of the cross coefficients and with the assigned values for parameters and coefficients. Due to the thermodynamic couplings hence the cross effects, there are excessive numbers of parameters controlling the behavior of temperatures and concentrations. Therefore, the results very much depend upon the magnitude and accuracy of the various parameters and coefficients used for internal and external parts of the system. The representative solutions are obtained based on several assumptions, such as equal diffusivities and surface concentrations for the components $S$ and $B$. Therefore, the results are representative and approximate, and based on the values of parameters listed in Table 1.

\section{Conclusions}

The balance equations are derived for thermodynamically and mathematically coupled heat and mass flows in a chemical reaction-transport system with external resistances to heat and mass flows. There are no thermodynamic couplings between chemical reaction and transport processes of heat and mass flows. These modeling equations are based on the linear nonequilibrium thermodynamics approach assuming that the system is in the vicinity of global equilibrium. They are capable of displaying the cross effects due to thermodynamic couplings on the mass compositions and temperatures as well as the effects of external resistances in time and space. The modeling equations have revealed some unique cross coefficients, which control thermodynamic couplings between the vectorial processes of heat and mass flows. These coefficients combine some measurable kinetic parameters, transport coefficients, and boundary values. Determinations of these coefficients may lead to a better understanding of the effects of thermodynamic couplings in reaction-transport phenomena.

\section{References}

[1] O. Aono, Thermodynamic coupling of diffusion with chemical reaction, J. Stat. Phys. 13 (1975), pp. 331-335.

[2] J. M. O. de Zarate, J. V. Sengers, D. Bedeaux, and S. Kjelstrup, Concentration fluctuations in non-isothermal reaction-diffusion systems, J. Chem. Phys. 127 (034501) (2007).

[3] Y. Demirel and S. I. Sandler, Linear non-equilibrium thermodynamics theory for coupled heat and mass transport, Int. J. Heat Mass Transfer 44 (2001), pp. 2439-2451.

[4] Y. Demirel, Non-isothermal reaction diffusion system with thermodynamically coupled heat and mass transfer, Chem. Eng. Sci. 61 (2006), pp. 3379-3385.
[5] Y. Demirel, Modeling of thermodynamically coupled reactiontransport systems, Chem. Eng. J. 139 (2008), pp. 106-117.

[6] Y. Demirel, Non-equilibrium Thermodynamics: Transport and Rate Processes in Physical, Chemical and Biological Systems (second ed.), Elsevier, Amsterdam (2007).

[7] P. Gas, C. Girardeaux, D. Mangelinck, and A. Portavoce, Reaction and diffusion at interfaces of micro- and nanostructured materials, Mater. Sci. Eng. B 101 (2003), pp. 43-48.

[8] J. V. Sengers and J. M. O. de Zarate, Thermal fluctuations in nonequilibrium thermodynamics, J. Non-Equilib. Thermodyn. 32 (2007), pp. 319-329.

[9] J. Xu, S. Kjelstrup, D. Bedeaux, and J.-M. Simon, Transport properties of $2 F=F 2$ in a temperature gradient as studied by molecular dynamics simulations, Phys. Chem. Chem. Phys. 9 (2007), pp. 969-981.

[10] G. Rambert, G. Jugla, J.-C. Grandidier, and L. Cangemi, A modeling of the direct coupling between heat transfer, mass transport, chemical reactions and mechanical behavior. Numerical implementation to explosive decompression, Compos. Part A 37 (2006), pp. 571-584.

[11] M. Li, Y. Wu, Y. Tian, and Y. Zhai, Non-thermal equilibrium model of the coupled heat and mass transfer in strong endothermic chemical reaction system of porous media, Int. J. Heat Mass Transfer 50 (2007), pp. 3936-3943.

[12] J. Yuan, F. Ren, and B. Sundén, Analysis of chemical-reaction-coupled mass and heat transport phenomena in a methane reformer duct for PEMFCs, Int. J. Heat Mass Transfer 50 (2007), pp. 687-701.

[13] A. M. Turing, The chemical basis of morphogenesis, Philos. Trans. R. Soc. Lond. B 237 (1952), pp. 37-72.

[14] R. S. Caplan and A. Essig, Bioenergetics and Linear Nonequilibrium Thermodynamics. The Steady State (Reprint), Harvard University Press, New York (1999).

[15] D. Kondepudi and I. Prigogine, Modern Thermodynamics; From Heat Engines to Dissipative Structures, Wiley, New York (1999).

[16] R. Aris, The Mathematical Theory of Diffusion and Reaction in Permeable Catalysts. Vol. 1: The Theory of the Steady State, Clarendon Press, Oxford (1975).

[17] F. G. Froment and K. B. Bischoff, Chemical Reactor Analysis and Design, Wiley, New York (1990).

[18] R. L. Rowley and M. D. Hall, Heat of transport from the diffusion thermoeffect in binary liquid mixtures of toluene, chlorobenzene, and bromobenzene, J. Chem. Phys. 85 (1986), pp. 3550-3555.

[19] S. C. Yi and R. L. Rowley, Measurement of heats of transport in ternary liquid mixtures via the diffusion thermoeffect, J. NonEquilib. Thermodyn. 14 (1989), pp. 293-297.

[20] G. Plat, T. Vongvanich, and R. L. Rowley, The diffusion thermoeffect in ternary nonelectrolyte liquid mixtures, J. Chem. Phys. 77 (1982), pp. 2113-2120.

[21] A. S. Cukrowski and A. Kolbus, On validity of linear phenomenological nonequilibrium thermodynamics equations in chemical kinetics, Acta Phys. Pol. B 36 (2005), pp. 1485-1507. 